\title{
Narrativa
}

\section{Maria Serena SAPEGNO, Figlie del padre. Passione e autorità nella letteratura occidentale, postfazione di Cristina Comencini}

\section{Serena Todesco}

\section{(2) OpenEdition}

\section{Journals}

Edizione digitale

URL: https://journals.openedition.org/narrativa/410

DOI: $10.4000 /$ narrativa.410

ISSN: 2804-1224

\section{Editore}

Presses universitaires de Paris Nanterre

\section{Edizione cartacea}

Data di pubblicazione: 1 décembre 2019

Paginazione: 180-182

ISBN: 978-2-84016-350-3

ISSN: $1166-3243$

Notizia bibliografica digitale

Serena Todesco, «Maria Serena sapegno, Figlie del padre. Passione e autorità nella letteratura occidentale, postfazione di Cristina Comencini», Narrativa [Online], 41 | 2019, online dal 01 novembre 2021, consultato il 08 décembre 2021. URL: http://journals.openedition.org/narrativa/410 ; DOI: https:// doi.org/10.4000/narrativa.410

Questo documento è stato generato automaticamente il 8 décembre 2021.

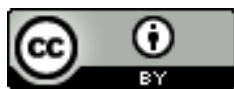

Narrativa est mise à disposition selon les termes de la Licence Creative Commons Attribution 4.0 International. 


\title{
Maria Serena SAPEGNO, Figlie del padre. Passione e autorità nella letteratura occidentale, postfazione di Cristina Comencini
}

\author{
Serena Todesco
}

\section{NOTIZIA}

Maria Serena SAPEGNO, Figlie del padre. Passione e autorità nella letteratura occidentale, Milano, Feltrinelli, 2018, postfazione di Cristina Comencini, 256 p.

1 Autrice di una lunga e originale attività di ricerca nel campo degli studi di genere, con importanti riflessioni su vari temi (dalla narrativa e poesia italiana antica, al sessismo linguistico e alla didattica universitaria in una prospettiva di genere), nel denso saggio Figlie del padre Maria Serena Sapegno declina poeticamente la propria capacità di creare e legittimare un dialogo tra generazioni. Il suo è un itinerario ricchissimo di letture provenienti da diverse tradizioni occidentali, dalla Bibbia al mondo classico di Omero e dei tragici greci, fino alla contemporaneità, con particolare attenzione dedicata al Settecento e all'Illuminismo, periodo che per Sapegno costituisce l'avvio di una liberazione simbolica delle figlie dalla Legge del Padre. Chiamate in causa sono qui le letterature di lingua inglese (Shakespeare, Virginia Woolf, Samuel Richardson, Fanny Burney, Jane Austen, sino a D. H. Lawrence, Sylvia Plath e Philip Roth), francese (Diderot, Madame de Staël, Zola, de Beauvoir), tedesca (Lessing, Schiller, Thomas Mann, Ingeborg Bachmann), e italiana, con incursioni nei mondi di Boccaccio, Metastasio, Alfieri, Goldoni, Verri, via via sciogliendo un fil rouge arricchito sempre più dalla presenza di voci femminili, da Amelia Rosselli a Clara Sereni, dalla Marchesa Colombi all'Artemisia di Anna Banti o alla Melania Mazzucco del romanzo La lunga attesa dell'angelo (2008). A dimostrare in filigrana il vivace corto circuito tra la tradizione e la 
modernità entro un discorso temporalmente trasversale è la frase tranchant che apre il saggio: "Certo noi non lo sapevamo, ma credo si possa dire che molte di noi non sarebbero approdate al movimento femminista senza i nostri padri", ribadendo così l'attualità di un'analisi del conflitto produttivo padre-figlia. Poco dopo Sapegno ne riassume lucidamente la contraddizione con parole che svelano l'atto autocoscienziale di una lettura/scrittura attenta a non scadere nelle idealizzazioni: "Erano loro, più che le nostre madri schiacciate tra due mondi, incerte e insoddisfatte, a invitarci più $o$ meno confusamente a metterci alla prova e a entrare pienamente nel mondo". Quel "noi non lo sapevamo" è un ponte semantico tra passato e presente, una lucida conversazione con le ragazze più giovani che hanno solo ascoltato, e non vissuto, il femminismo e il '68. La testimonianza di Sapegno, conscia del portato psicologico e storico della relazione tra i padri e le figlie nella cultura e nella letteratura italiane e occidentali, consente proprio alla generazione delle "figlie" oggi ventenni o trentaquarantenni di capire le ragioni dietro le fratture della società, oggi come cinquant'anni fa. Sapegno rilegge criticamente i rapporti tra le donne e lo spazio pubblico proprio alla luce della necessità di guardare ai padri, prima ancora che alle madri, storicamente escluse dal discorso culturale dominante. Proprio nonostante i padri, ci dice, non avremmo potuto compiere il passo decisivo, non avremmo pasolinianamente "gettato il proprio corpo nella lotta". E i corpi sono quelli di figlie che scrivono, più o meno solitarie nella propria battaglia per l'autonomia artistica ed esistenziale. Con stile fluido e insieme denso e consapevole, il saggio di Sapegno intesse allora molteplici dialoghi tra i padri e le figlie della letteratura occidentale, riportando alla memoria collettiva dei lettori l'idea che il padre, a differenza della madre, compaia regolarmente nelle vicende individuali della vita di numerosissime donne, siano esse "personagge" o scrittrici. Nel volgere lo sguardo all'indietro, "agli archetipi che la modernità rilegge e da cui è ossessionata", la presenza del padre rappresenta infatti una soglia di dolore, di crescita e di affrancamento progressivo o mancato, da cui può scaturire una consapevolezza che conduce alla scrittura, alla pratica artistica. Dalla lettura del saggio di Sapegno si intuisce come la condizione di "figlie del padre" si perpetui causando un effetto duplice, fondamentale per comprendere non soltanto l'ambivalenza del conflitto stesso, ma anche quel depotenziamento della mascolinità di cui tanto si discute nell'attuale dibattito socio-culturale. In quest'ottica, allora, Sapegno indirettamente suggerisce che l'acquisizione di soggettività delle donne tramite la scrittura non può essere esclusivamente letta attraverso il filtro dell'emancipazione, né a partire dal binarismo oppositivo dei sessi in lotta perenne: come per le figlie di tanti padri della letteratura, talvolta divenute scrittrici consapevoli di una propria voce, quella ribellione, rivissuta dalle ragazze del femminismo novecentesco, non tende a sostituirsi alla Legge del Padre, piuttosto si condensa in una contraddizione più profonda, bruciante, continuamente da rinegoziare, ovvero la coesistenza problematica tra il desiderio di cambiamento e di legittimazione e, d'altra parte, l'urgenza di non tradire mai la propria unicità. 